\title{
Markus Frankl, Martina Hartmann (dir.), Herbipolis. Studien zu Stadt und Hochstift Würzburg in Spätmittelalter und früher Neuzeit
}

Jean-Dominique Delle Luche

\section{OpenEdition}

Édition électronique

URL : http://journals.openedition.org/ifha/8740

DOI : $10.4000 /$ ifha. 8740

ISSN : $2198-8943$

\section{Éditeur}

IFRA - Institut franco-allemand (sciences historiques et sociales)

\section{Référence électronique}

Jean-Dominique Delle Luche, « Markus Frankl, Martina Hartmann (dir.), Herbipolis. Studien zu Stadt und Hochstift Würzburg in Spätmittelalter und früher Neuzeit », Revue de l'IFHA [En ligne], Date de recension, mis en ligne le 02 novembre 2017, consulté le 24 septembre 2020. URL : http:// journals.openedition.org/ifha/8740; DOI : https://doi.org/10.4000/ifha.8740

Ce document a été généré automatiquement le 24 septembre 2020.

(C)IFHA 
Markus Frankl, Martina Hartmann (dir.), Herbipolis. Studien zu Stadt und Hochstift Würzburg in Spätmittelalter und früher Neuzeit

Jean-Dominique Delle Luche

\section{RÉFÉRENCE}

Markus Frankl, Martina Hartmann (dir.), Herbipolis. Studien zu Stadt und Hochstift Würzburg in Spätmittelalter und früher Neuzeit, Würzburg: Königshausen und Neumann, 2015, 482 p., $39,80 €$ 
Ce volume rassemble 18 contributions de taille inégale (trois articles représentent à eux seuls 150 des 450 pages de texte) en l'honneur de Franz Fuchs, titulaire de la chaire d'histoire médiévale de l'université de Wurtzbourg depuis 2002. La majorité des articles est consacrée à l'histoire régionale de la ville et du diocèse de Wurtzbourg entre le $\mathrm{XI}^{\mathrm{e}}$ et le $\mathrm{XVI}^{\mathrm{e}}$ siècle. Les thèmes et les échelles sont caractéristiques des inflexions thématiques de la Landesgeschichte, passant de l'histoire impériale pour le Moyen Âge central à une histoire régionale, locale ou culturelle pour les $\mathrm{XV}^{\mathrm{e}}$ et $\mathrm{XVI}^{\mathrm{e}}$ siècles.

C. Ehlers trace un parallèle entre Francfort et Wurtzbourg, deux villes caractéristiques de la politique

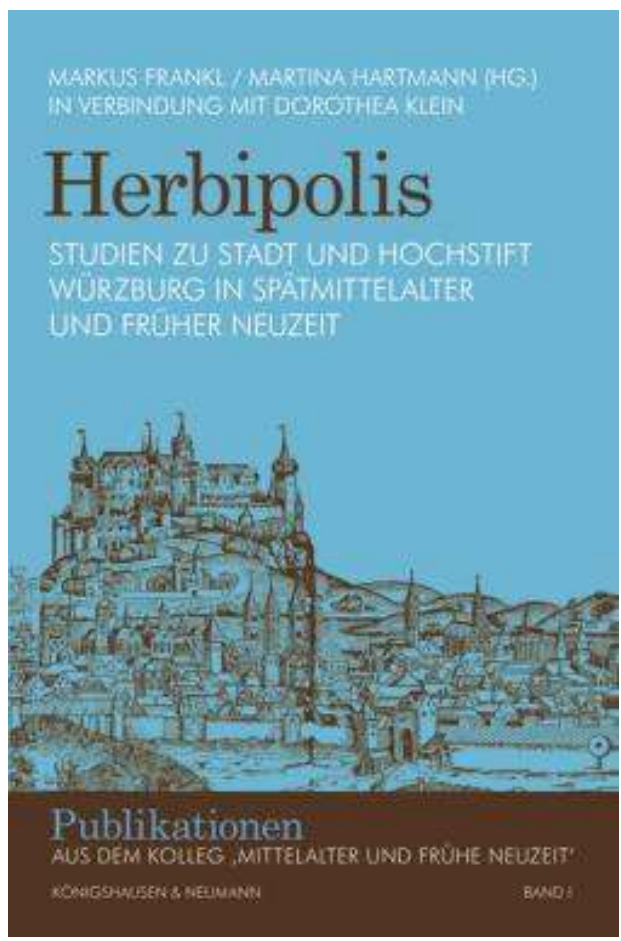
territoriale carolingienne; il montre que, si l'une est liée au pouvoir impérial et l'autre marquée par l'évêque, les fonctions des deux villes tendent à se confondre sous les Staufen. Paradoxalement, Francfort accueille moins souvent les souverains que Wurtzbourg, dont l'évêque obtient le titre recherché de duc de Franconie. Cet article est étonnamment placé après celui de K. Borchardt, qui concerne pourtant une chronologie postérieure et réexamine la question des ministériaux au service des Hohenstaufen dans la région. Les empereurs font venir les sénéchaux (Truchsess) de Rothenbourg depuis la Suisse actuelle pour sécuriser le sud de la Franconie, avant de les remplacer par deux familles successives qui obtiennent l'office impérial nouvellement créé de maître des cuisines (Reichsküchenmeister). Les crises du pouvoir impérial (règne de Philippe de Souabe, révolte de Henri (VII)) éclairent les revers et les progressions de l'aristocratie et des ministériaux. Menée prudemment, la reconstitution de la généalogie (y compris les liens hypothétiques avec le célèbre Minnesänger Walther von der Vogelweide) souligne l'intérêt de réexaminer l'héraldique et l'archéologie pour compléter les informations fournies par les diplômes.

Le $\mathrm{XIV}^{\mathrm{e}}$ siècle est classiquement celui où les empereurs alternent entre une politique favorable et une hostilité affichée aux villes: U. Wagner édite et présente deux privilèges conférés à Wurtzbourg par l'empereur Louis de Bavière, et $\mathrm{M}$. Naser narre le dernier engagement de Rothenbourg ob der Tauber sous Charles IV. Cette contribution éclaire les conflits au sein de la bourgeoisie, partagée entre l'empereur et l'évêque de Wurtzbourg. Avec Heidingsfeld (C. Groth), entre 1455 et 1457, c'est encore une fois la situation singulière d'une ville d'Empire engagée qui est évoquée, à travers une révolte du corps de bourgeoisie contre ses engagistes. De nouveaux acteurs sont évoqués : les margraves de Brandebourg-Ansbach (qu'on retrouve avec E. Bünz), la ville de Nuremberg et le roi de Bohême.

On revient au territoire du prince-évêque de Wurtzbourg avec les quatre contributions suivantes. S. Petersen reconstitue les différentes stratégies d'exploitation du bailliage 
de Gerolzhofen, régulièrement engagé jusqu'à un rachat nécessaire dans le cadre d'un approfondissement du pouvoir territorial et d'une situation budgétaire délicate. $\mathrm{Ce}$ même pouvoir territorial est analysé à travers les conflits juridiques avec les comtes de Wertheim (F. Kleinehagenbrock) puis la gestion d'un monastère de cisterciennes, l'évêque rivalisant avec des nobles locaux. L'inscription votive dédiée à sainte Barbara (E. Bünz) par la ville d'Ochsenfurt rentre dans le cadre de la mémoire des conflits en ville (ici, une surprise de nuit). M. Frankl consacre plusieurs dizaines de pages aux enjeux économiques et politiques de la viticulture à Wurtzbourg, encore très forte dans le paysage actuel de la capitale régionale.

Dans la deuxième moitié de l'ouvrage, on trouvera davantage de contributions concernant l'histoire artistique et littéraire de Wurtzbourg, à travers l'étude des tropes (H. Zühlke), de la poésie d'actualité (H. Brunner), des représentations de la citadelle de Wurtzbourg dans la chronique de Hartmann Schedel (S. Kummers). L'étude d'une brève correspondance amoureuse conservée à Ratisbonne est un peu hors sujet, même si B. Lübbers suppose une provenance lointainement wurtzbourgeoise. Les dernières contributions s'éloignent progressivement de l'espace wurtzbourgeois pour se recentrer sur les thèmes de recherche de Franz Fuchs, en particulier l'histoire des bibliothèques et des humanistes. Pas moins de trois articles (K. Arnold, A. MentzelReuters et M. Hartmann) tournent autour de la personne de l'humaniste Trithemius et de ses écrits historiques. M. Hartmann présente le destin d'une dizaine de bibliothèques de lettrés, s'interrogeant sur les "chances de transmission » de celles-ci et pointant souvent une stratégie fatale, la transmission aux descendants ou à des privés, tandis que le legs à une église ou à la ville réduit la dispersion des collections. Le dernier article (F. Kahle) examine la correspondance du Nurembergeois Christoph Scheurl avec ses partenaires de Wurtzbourg.

Certains articles se retrouvent dans des publications parallèles, mais dans l'ensemble les contributions correspondent aux standards de qualité de l'histoire régionale érudite allemande. Le lecteur français sera parfois désorienté par des contributions cachant les idées fortes derrière l'exposé chronologique ou analytique rigoureux. On saluera, outre un index fourni des noms et des lieux, les nombreuses illustrations du volume, qu'il s'agisse de l'iconographie, d'arbres généalogiques, de louables reproductions de manuscrits ainsi que la présence de cartes, toujours trop rares dans les publications allemandes, mais fréquentes à l'université de Wurtzbourg. Dans le tour d'horizon géographique assez conséquent (remettant en valeur des territoires ou des localités comme Wertheim, Heidingsfeld, Gerolzhofen ou Ochsenfurt), on regrettera que le nord du diocèse, avec la Thuringe méridionale, ou le territoire voisin de l'évêché de Bamberg ne soient pas évoqués : l'ouvrage se contente de la Basse-Franconie telle qu'elle existe dans les frontières politiques actuelles, sans chercher à aller au-delà des limites traditionnelles ou contemporaines de la Landesgeschichte wurtzbourgeoise. 
INDEX

Index chronologique : Moyen Âge, Période moderne

Thèmes : Histoire des villes et des régions

\section{AUTEURS}

JEAN-DOMINIQUE DELLE LUCHE

EHESS 